\title{
ROADMAP FOR SECURING HANDHELD DEVICES
}

\section{System Integrity and Confidentiality}

\author{
Preetida Vinayakray-Jani \\ Nokia Research Center \\ P.O.BOX 407, FIN-0004.5 NOKIA GROUP, Finland \\ preetida.vinayakray-jani@nokia.com
}

\begin{abstract}
With increasing connectivity and data sharing possibilities with handheld devices, potential threats to device security, user privacy and home/corporate LAN have become very significant. Currently there is no prevention against malicious software and supported access control - if any at all - is lacking any security relevant attributes. Often when such countermeasures are designed, the security is traded for performance and battery power. Key security issues within handheld devices necessitate the mandatory security policies to be assigned and enforced. This paper creates the roadmap for securing handheld devices by identifying security relevant policies and discusses how such policies can be assigned and enforced '.
\end{abstract}

Keywords: Malicious software, System security, Software development and execution

\section{Introduction}

While user takes an advantage of handheld devices with multi-interfaces e.g. WLAN, Bluetooth, UMTS etc. and increased computational power, there are significant security risks posing threats to user privacy, integrity of the device and network. Versatility of this device allows users to send and receive emails, to access corporate data, to surf Internet, and to sync and transfer stored data to home PC or PC within corporate LAN. Eventually when handheld devices due to their flexibility, compactness and usefulness have become prevalent in corporate environment, the encountered security risks include:

- Wireless Interception: Connecting handheld devices to vulnerable web sites, receiving/transmitting unencrypted emails or usage of flawed cryptographic tools will allow an attacker to create Man-in-the middle attack.

\footnotetext{
'Disclaimer: The views expressed are those of the author and do not represent an official view of Nokia Research Center.
}

The original version of this chapter was revised: The copyright line was incorrect. This has been corrected. The Erratum to this chapter is available at DOI: 10.1007/978-0-387-35691-4_52 
- Probing: Probing in sensitive area and directing confidential information to unauthorized users can generate privacy threats to user/corporation.

- Malicious Software/Code: Installation of software without verifying its integrity and trustworthiness. Often this practice leads to malware ${ }^{2}$ attack to home/corporate network.

- Weak/Null Authentication: Used authentication mechanism is either weak or disabled, allowing access to unauthorized users.

- Roaming in dangerous environment: Any device roaming in vicinity of the other handheld device can create intentional attack and deprive particular handheld device from providing/receiving any service.

- Lack of user initiative and awareness: Very frequent users do not take strong initiative to protect information stored within device. Also sometimes user does not recognize that sensitive information stored within the device is vulnerable and about to be compromised by the attacker.

Mitigation of such risks, through tools (e.g. antivirus, sandboxing, access control etc.) is unable to keep pace with the emerging new applications. Compared to conventional applications, current applications draw upon the services and resources from other applications and not just the operating system [1]. In fact, the successful impact of application specific security solutions very much hinges over the secure platform. Therefore, when system platforms of handheld devices are designed to be open and modular to support third party applications, the integrity and confidentiality of system as well as software applications cannot be overlooked.

Hence in this paper section 2 discusses the platform security including its inadequacy against malicious code and policies necessary to derive secure system for device. Section 3 examines existing software flaws in the application in terms of buffer overflow, lack of memory protection, system stability and mobile code. Section 4 provides conceptual framework for assigning and enforcing the security policies. Finally section 5 presents the concluding remarks.

\section{Platform Security}

Since the handheld devices are extended with capability like remote connectivity or access, the span of its authorization has also expanded; a malicious program can cause damage to any installed application, except that there is no authorization. Malicious software can easily override the user's access rights

\footnotetext{
${ }^{2}$ This includes viruses, Trojans and worms. Together they are also called malware.
} 
for any particular objects, making malicious application indistinct from legitimate one. With lack of user authorization role it is difficult to identify which activities are permissible or not and this can lead to all types of attacks. Since there is no strong coupling between user and the code he executes, current handheld device platforms neither support integrity of the code nor provide logical means to classify roles of users so that sensitive information can be protected.

This can be prevented if the authorization is outside the control of typical user. This can be achieved by means of mandatory security policies in which authority is vested in some users - system administrators, but there are control on delegation and allocation of authority. Research efforts made by [3] have clearly emphasized the usage of mandatory security policy, classified into various kinds of policies such as:

- Authentication policy for users to prevent unauthorized access

- Differentiating policy for users with specific functional roles (e.g. security administrator for system and ordinary user) and process privileges

- Authorization policy for accessing confidential information and database

- Integrity policy for file access to prevent tampering of data/application

Any possible adversary action to invoke malicious software can override the access mechanism policies of the files belonging to the authorized user. Thus access control mechanism also requires the trusted path mechanism and that will allow authorized user to access trusted software only [3]. The practical means of assigning mandatory policy should be justified through suitable mechanisms where policy can be enforced with consistent cost.

\section{Software flaws in Application: Development, Instillation and Execution}

Today when evolution of system is reliant on applications, attackers are looking for gaining unauthorized access on system by exploiting vulnerabilities in application program through buffer overflow, depriving user from using any particular application or passing dangerous command to system subroutines.

\section{Buffer Overflow}

Sometimes the system vulnerability is exposed to the flaws in standard software coding techniques. Nonetheless, this vulnerability may be due to the inadequacy of the system or it may be inherent to coding method. Very frequent application developer is careless about examining the size of the arguments and 
stores them into a buffer of fixed size using standard functions causing buffer overflow. Application with buffer vulnerability often gives attacker an opportunity to inject and execute malicious software. An effective coding language and method should be used to prevent buffer overflow.

\section{Lack of Memory Protection}

With current device system all application/data reside within battery-backed RAM. It is very likely that residing bad applications stomp on themselves and also on other trusted/untrusted applications or the system itself. Encountered error in one application can reflect erroneous changes in subsequent trusted/untrusted application, eventually crashing system itself. Thus memory protection is needed for preserving integrity and privacy of applications. Implementing hardware-based memory management unit (MMU) in conjunction with secure and reliable bootstrap architecture is one possible solution to this problem as usage of ROM and Flash memory is not convincing [5].

\section{System stability}

Even if memory protection is encountered efficiently there are still risks if the command used to pass on malicious parameters to system subroutines. With this system does not know that it has been asked to do something that is pernicious. Since system does not access to the application memory, it is possible to break the system integrity without violating memory protection. Therefore mandatory policy like sanity checking is needed to verify parameters before executing them.

\section{Mobile Code: Automate Execution}

Another possible risk is the containment of untrusted mobile code. Neutral to any operating platform this code can be distributed seamlessly over Internet and is often received by a device with request to execute with user privileges. Apparently this automatic execution does not postulate any existing vulnerability, as system does not acquire such mechanisms evaluating the security implications of the executing code. Any failure to handle this code result in more serious damage like giving significant privilege to access sensitive information and system resources, creating denial of service (DoS) attack or penetrating in corporate LAN through downloaded Trojan or virus [2]. Although policy like implementing sandboxes can be useful to constrain mobile code but that is not enough as any implementation errors enable mobile code to circumvent the sandbox's security mechanisms. Solution like Program shepherding can be applicable but that also requires the un-circumventable sandboxing [6]. In brief, proper mechanisms are needed for system to prevent the harmful influence of the mobile code. 


\section{Security Administration: A Conceptual Framework}

Having all mentioned above the risk mitigation through efficient security measures is not panacea for future security risks that are not feasible today. Despite the fact that future handheld device might be governed through the proper security measures, the corporate LAN will remain on the verge of security threats if prescribed security of corporate is bypassed. As a result following discussion provides the conceptual view of assigning security policy and enforcing it. A very similar but detailed approach proposed as a proof-ofconcept in [4] for Palm OS. Over here proposed work does not emphasize any particular operating platform of handheld device.

\section{Assignment of Proprietary Policy}

Handheld device should contain the default security policy as well as proprietary policy assigned by the corporate system administrator. Default policies are rudimentary that confine the usage of device for a very limited applications. Proprietary Policy assigned by security administrator should handle the usage of assets and audit log of the device. Here asset means the confidential information specific to corporation/user. Policy should assign security attributes with program that will support system security configuration management and protect against malicious modification of program by malware. Also policy should assign other security attributes associated with program that will restrict specific operation permitted by specified program on specified objects when they are executed.

To maintain the integrity of the device against new attacks, assigned proprietary policies should be frequently updated. Moreover, assigned proprietary policies should be protected by means of theft protected proprietary certificate that can be validated by means of suitable mechanism.

\section{Enforcement of Proprietary Policy}

The policy enforcement mechanism on handheld device should be configured according to the assigned proprietary policies and its activation assures security administrator that device as well as user has complied with the assigned proprietary policies. Once device validates the proprietary policy certificate policy enforcement mechanism should monitor and govern any security relevant activities on device.

Enforced policies should update the audit log and restrict the external flow of the confidential information. The audit log should store fine grain (memory access) security events to high-level operations (process calls) to the operation that can change the security configurations. Enforcement mechanism may contain many mechanisms in terms of access control, installation of third party 
software, periodic syncing with the network, determent of theft etc. Monitoring and managing the periodic sync of audit log information on computer allows security administrator to analyze the collected log and take necessary actions if any anomalies are found.

\section{Conclusion}

This paper addresses the inadequacy of the current handheld devices against the malicious code. Privacy and integrity of the device not only requires the secure platform but also efficient software engineering and memory management. To gain acceptance of handheld device as Trusted Device in corporation or in enterprise a conceptual framework for assigning and enforcing the proprietary policies is presented. Overall paper provides the roadmap for securing handheld device stemming from the platform security to assignment and enforcement of proprietary policies.

\section{Acknowledgement}

Author is grateful to Markku Kylänpää and Kaisa Nyberg for providing helpful comments and feedback, Jani Suomalainen for updating and discussing mandatory security approaches, and Janne Mäntylä for supporting this work.

\section{References}

[1] Marshall D. Abrams and Michael V. Joyce. (1995). Trusted System Concept. Computers \& security, Vol. 14 No.1, Pages 45-56, Copyright Elsevier Advanced Technology 1995. Oxford, UK.

[2] Matt Blaze, Joan Feigenbaum, John Ioannidis and Angelos D. Keromytis. (1999). The Role of Trust Management in Distributed System Security. Book: Secure Internet Programming: Security Issues for Mobile and Distributed Objects, ed. Jan Vitek and Christian Jensen, Springer-Verlag Inc., New York, NY, USA. Pages 185-210. 1999.

[3] Peter Loscocco, Stephen Smalley. (2001). Integrating Flexible Support for Security Policies into the Linux operating system. Technical Report, NSA and NAI Labs, February 2001.

[4] Wayne Jansen, Tom Karygiannis, Serban Gavrila, and Vlad Korolev. (2002). Assigning and Enforcing Security Policies on Handheld Devices. In Proceedings of the Canadian Information Technology Security Symposium, May 2002.

[5] Kingpin and Mudge. (2001). Security Analysis of the Palm Operating System and its Weakness Against Malicious Code Threats. In Proceedings of the 10th USENIX Security Symposium, Washington, DC, August 13-17, 2001, Pages 135-15I, ISBN 1-880446-07-3.

[6] Vladmir Kiriansky, Derek Bruening and Saman Amarasinghe. (2002). Secure execution Via Program Shepherding. In Proceedings of the 11 th USENIX Security Symposium (Security '02), San Fransisco, California, August 2002. 